\title{
Die TYPHOON-Studie
}

\section{Trial to assess the use of the cYPHer stent in acute myocardial infarction treated with ballOON angioplasty}

Hintergrund und Problemstellung: Die primäre PCI ist die beste Therapie des akuten Myokardinfarktes mit STStrecken-Hebung (STEMI). Die Implantation eines unbeschichteten Stents (BMS) sichert den Akuterfolg, führt allerdings zur späteren In-Stent-Restenose. In den größeren Studien mit

\begin{tabular}{ll}
\hline Methodik & \\
\hline Studiendesign & Randomisiert, kontrolliert \\
Primärer Endpunkt & $\begin{array}{l}\text { TVF (target vessel failure) nach 1 Jahr (defi- } \\
\text { niert als ischämieindizierte TVR, Reinfarkt, } \\
\text { TV-bezogener Tod) } \\
\text { Einschlusskriterien: Patienten mit STEMI < 12 h, } \\
\text { die eine primäre PCI benötigen; ST-Hebung } \\
\geq 1 \text { mm in } \geq 2 \text { Ableitungen oder neu } \\
\text { aufgetretener Linksschenkelblock } \\
\text { Ausschlusskriterien: aktuelle Fibrinolyse, } \\
\text { Killip-Klasse }>2, \text { vorausgegangener MI, vor- } \\
\text { ausgegangene PCI am Zielgefäß, LVEF <30\%, } \\
\text { Niereninsuffizienz, ostiale Stenosen, Bifurkati- } \\
\text { onsstenosen, starke Kalzifizierung/Schänge- } \\
\text { lung, massive Thrombuslast } \\
48\end{array}$ \\
$\begin{array}{ll}\text { Anzahl der teil- } \\
\text { nehmenden Zentren }\end{array}$ &
\end{tabular}

Ergebnisse $(* p<0,05)$

\begin{tabular}{|c|c|c|}
\hline & $\begin{array}{l}\text { Kontrollgruppe: } \\
\text { (jeglicher BMS) }\end{array}$ & $\begin{array}{l}\text { Cypher/ } \\
\text { Cypher Select }\end{array}$ \\
\hline Patientenzahl & $\mathrm{n}=357$ & $\mathrm{n}=355$ \\
\hline $\begin{array}{l}\text { Patienten- } \\
\text { charakteristika }\end{array}$ & \multicolumn{2}{|c|}{$\begin{array}{l}\text { Beide Gruppen hinsichtlich der Ausgangs- } \\
\text { parameter vergleichbar, jedoch signifikante } \\
\text { Unterschiede hinsichtlich Patientenalter (BMS: } \\
60,5 \pm 12,4 \text {; DES: } 58,0 \pm 11,8 \text { ) und Anzahl der } \\
\text { vorausgegangenen PCIs (BMS: } 5,9 \text {; DES: } 2,5 \text { ), } \\
\text { max. Stentdurchmesser (BMS:3,1 } 10,4 ; \text { DES: } \\
3,0 \pm 0,3 \mathrm{~mm} \text { ) und Stentlänge (BMS: 20,2 } \pm \\
\text { 8,2; DES: } 21,1 \pm 8,6 \text { ) }\end{array}$} \\
\hline $\begin{array}{l}\text { Primärer Endpunkt } \\
\text { (s. o.) } \\
\text { Angiographische } \\
\text { Parameter (8 Monate): }\end{array}$ & $14,3 \%$ & $7,3 \%$ * \\
\hline $\begin{array}{l}\text { Binäre Restenoserate } \\
\text { in Stent }\end{array}$ & $20,3 \%$ & $3,5 \%$ * \\
\hline $\begin{array}{l}\text { Late Loss in Stent } \\
\text { nach } 8 \text { Monaten } \\
\text { (Subgruppe } 200 \text { Patienten) }\end{array}$ & $0,83 \pm 0,52 \mathrm{~mm}$ & $0,13 \pm 0,49 \mathrm{~mm}^{*}$ \\
\hline $\begin{array}{l}\text { Klinische Parameter } \\
\text { (12 Monate): }\end{array}$ & & \\
\hline Tod & $2,2 \%$ & $2,2 \%$ \\
\hline Gesamte Myokardinfarkte & $1,4 \%$ & $1,1 \%$ \\
\hline Gesamte Stentthrombosen & $3,6 \%$ & $3,4 \%$ \\
\hline $\begin{array}{l}\text { TVR (target vessel } \\
\text { revascularization) }\end{array}$ & $13,4 \%$ & $5,6 \%$ \\
\hline MACE & $14,6 \%$ & $5,9 \%$ * \\
\hline
\end{tabular}

Medikamente freisetzenden Stents (DES) wurden Patienten mit STEMI ausgeschlossen. Ziel der Studie war der Nachweis von Wirksamkeit und Sicherheit des Cypher-Stents im Vergleich zu BMS bei Patienten mit STEMI. Studienleiter waren C. Spaulding, Paris, Frankreich und C. Bode, Freiburg.

\begin{tabular}{|c|c|c|}
\hline Klinischer primärer Endpunkt & $\mathrm{Ja}=3$, nein $=0$ & 3 \\
\hline Doppelblind (einschließlich Ärzten) & $\mathrm{Ja}=1$, nein $=0$ & 0 \\
\hline $\begin{array}{l}\text { Beobachtungsintervall } \\
\text { für den primären } \\
\text { Endpunkt } \geq 6 \text { Monate }\end{array}$ & $\mathrm{Ja}=1$, nein $=0$ & 1 \\
\hline Multicenter (mindestens 3 Zentren) & $\mathrm{Ja}=1$, nein $=0$ & 1 \\
\hline $\begin{array}{l}\text { Externes und vom } \\
\text { Steering Committee unab- } \\
\text { hängiges Clinical Event } \\
\text { Committee / DSMB } \\
\text { (Datensicherheit } \\
\text { Monitoring Board) }\end{array}$ & $\mathrm{Ja}=1$, nein $=0$ & 1 \\
\hline Primärer Endpunkt erreicht & $\mathrm{Ja}=1$, nein $=0$ & 1 \\
\hline $\begin{array}{l}\text { Power von } \geq 80 \% \text { für den } \\
\text { primären Endpunkt erreicht }\end{array}$ & $\mathrm{Ja}=1$, nein $=0$ & 1 \\
\hline $\begin{array}{l}\text { Anteil des Follow-up der Patienten } \\
\text { für angiographischen primären } \\
\text { Endpunkt } \geq 80 \% \text { und } \geq 95 \% \text { für } \\
\text { klinischen primären Endpunkt }\end{array}$ & $\mathrm{Ja}=1$, nein $=0$ & 1 \\
\hline \multicolumn{2}{|c|}{$\begin{array}{l}\text { Gesamt-EBM-Score } \\
\text { („Silber-Score“ für randomisierte Studien) }\end{array}$} & 9 \\
\hline
\end{tabular}

Schlussfolgerung und Kommentar: TYPHOON ist die erste randomisierte Multicenterstudie, die anhand eines primären klinischen Endpunkts den Cypher-Stent mit BMS bei STEMI-Patienten verglich. Der primäre Endpunkt (TVF nach 1 Jahr) wurde erreicht. Die Reduktion des TVF war entscheidend von der Abnahme der klinisch indizierten Re-PCI bestimmt. Limitierend ist zu bedenken, dass die nach 8 Monaten routinemäßig durchgeführte Kontrollangiographie das Ergebnis nach 12 Monaten entscheidend beeinflusst haben könnte. Das Ausmaß der Reduktion des Late Loss war im Bereich von Patienten mit stabiler KHK. Die Rate an Stentthrombosen war in beiden Gruppen gleich - aber mit $>3 \%$ auffallend hoch. Dies mag mit der neuen Definition der Stentthrombose erklärt werden, in die jeglicher (!) Herztod innerhalb der ersten 30 Tage einging.

Herz 2006;31:169

DOI $10.1007 / \mathrm{s} 00059-006-2811-0$ 